ISSN 0258-7122

Bangladesh J. Agril. Res. 39(3): 461-469, September 2014

\title{
EFFECT OF RELATIVE HUMIDITY, INITIAL SEED MOISTURE CONTENT AND STORAGE CONTAINER ON SOYBEAN (Glycine max L. Meril.) SEED QUALITY
}

\author{
M. R. ALI ${ }^{1}$, M. M. RAHMAN ${ }^{2}$ AND K. U. AHAMMAD ${ }^{3}$
}

\begin{abstract}
To find out the effect of storage relative humidity, seed moisture content and type of storage container on soybean seed quality, an experiment was conducted at the Seed Laboratory, Department of Agronomy, Bangladesh Agricultural University Mymensingh in 2008 and 2009. In 2008, soybean seed has 96\% initial germination and in 2009 seed having $98 \%$ initial germination was stored at $8 \%$ and $12 \%$ initial moisture levels in two types of storage containers viz., cloth bag and polythene bag $(0.06 \mathrm{~mm}$ thickness). The final seed moisture content, germination percentage, germination index, and seedling dry matter of the seed under different treatments were measured at 60,120, and 180 days after storage (DAS). The experiment was arranged in a completely randomized design with three replications. In 2008, highest germination percentage $(89.33 \%)$ of soybean seed was retained at 180 days after storage (DAS) for those stored at $8 \%$ initial seed moisture content (SMC) in polythene bag at $50 \%$ relative humidity. Germination index and seedling dry matter decreased with increased initial seed moisture content irrespective of storage containers used. In 2009 , highest germination percentage $(92.67 \%)$ of soybean seed was retained at 180 DAS for those stored in polythene bag at $8 \%$ initial SMC at $50 \%$ of the relative humidity. Those stored in cloth bag at $12 \%$ SMC showed rapid germination loss and the value went down to 0.00 in both the years. Vigour index and seedling dry matter decreased with increased initial seed moisture content irrespective of storage containers used.
\end{abstract}

Keywords: Soybean, relative humidity, seed moisture, container, viability, vigour.

\section{Introduction}

The problems of maintaining the soybean seed viability in storage have always been an important concern; and retention of high viability over a long period is necessary for crop production. Many factors determine the longevity of seeds during storage. These includes seed moisture content, temperature, relative humidity, initial viability, stage of maturity at harvest, storage gas and initial moisture content of seed entering into storage (Tatipata, 2009). Soybean seed losses its viability in very short period of storage even when stored in good nonporous container (Woodruff, 1998). The seeds with low moisture content and

${ }^{1}$ Senior Scientific Officer, RARS, Bangladesh Agricultural Research Institute (BARI), Jamalpur, ${ }^{2}$ Professor, Department of Agronomy, Bangladesh Agricultural University (BAU), Mymensingh, ${ }^{3}$ Senior Scientific Officer, RARS, BARI, Jessore, Bangladesh. 
stored in any air tight containers could retain viability for a longer period of time. Sealed plastic pot and polythene bag are more effective storage containers than cloth bag or jute bag and earthen pot etc. (Rahman et al., 2010). Soybean seed is rapidly deteriorated by high temperature and high relative humidity during storage (McDonald, 1999). Several factors may affect the quality of seeds in storage, however, the most critical among them is high seed moisture content (O'Hare et al., 2001). High moisture content and presence of oxygen were the main causes for lipids autoxidation in soybean to lead rapidly seed deterioration and the quality decline (Chang, 2004). Seed must be dried to a safe moisture level immediately after harvest and it should be maintained until planting. Seeds stored at low moisture content or at low relative humidity (RH) retain viability for longer period (Miah, 2006). If seed packaged in moisture proof containers and the relative humidity of the air around the seed remains low, then the seed equilibrium moisture remains low and the seed maintains its viability and vigour for a longer time (Agha et al., 2004). Thus it appears that seed being stored in low relative humidity at lower moisture content in air tight containers could retain high viability for a long period of time. However, research work regarding the effect of relative humidity, initial seed moisture content and storage container on soybean seed quality is scarce. Therefore, an experiment was undertaken to find out the effect of relative humidity, initial seed moisture content and storage container on soybean seed quality.

\section{Materials and Method}

The experiment was conducted at the Seed Laboratory of the Department of Agronomy, Bangladesh Agricultural University, Mymensingh during May to November 2008 and 2009. The seeds were stored at four different levels of relative humidity viz., $50,60,70$, and $80 \%$ in polythene bag and cloth bag with 8.0 and $12.0 \%$ initial moisture content. The treatments were arranged in Completely Randomized Design with three replications. The soybean variety Shohag ( $\mathrm{Pb}$ 1) were collected from Regional Agricultural Research Station, BARI, Jamalpur. Relative humidity in the storage (cleaned and sterilized glass jar of $28.0 \mathrm{~cm}$ diameter and $35.6 \mathrm{~cm}$ height) were created using table salt $\left(\mathrm{NaCl}_{2}\right)$ and water at different ratios. The salt $(\mathrm{g})$ and water $(\mathrm{ml})$ in the ratio 200:0, 100:0, 50:50, and 25:75 to create 50,60, 70, and $80 \% \mathrm{RH}$ were taken in the glass jar. Five hundred grams of seeds were taken in each of cloth or polythene bags. Samples were taken at 60 days interval for 180 days. The relative humidity of storage container was measured with the hygrow meter. The moisture content, germination percentages, germination index and seedling dry matter were determined.

Seed moisture content: Seed moisture content was measured using high constant temperature oven dry method following ISTA rules (2003). About 5-8 g of soybean seeds were placed in aluminium dish and dried in oven at $130{ }^{\circ} \mathrm{C}$ for 2 
hours (until constant weight achieved). Then the sample was cooled in a desecrator and weighed. Then the moisture content was calculated as follows:

Moisture content $(\%)=\frac{W 1-W}{W 1-W 2} \times 100$

Where, $\mathrm{W}=$ Weight of blank aluminium dish with lid

$\mathrm{W}_{1}=$ Weight of seed plus aluminium dish with lid before drying

$\mathrm{W}_{2}=$ Weight of seed plus aluminium dish with lid after drying

Germination test: Germination test of seed was done in sand petridish method using sterilized sand. Randomly collected 50 seeds in three replicates per bag were used in the test. The sand was moistened to $60 \%$ water holding capacity before placing it on the petridish. Seed germination evaluation was done at 8 days after placing the test. The number of normal seedlings, abnormal seedlings and dead seeds were recorded respectively as per ISTA rules (2003). The sum of three replicates per bag was used and the germination was expressed in percentage.

Germination $(\%)=\frac{\text { Number of seeds germinated }}{\text { Number of seeds sown }} \times 100$

Germination index (GI): Germination index of seed was estimated from the seed set in the germination test by calculating the germination index following the formula below given by Association of Official Seed Analysts (AOSA, 1983). The number of seedling was counted at each day at the same time from the day after seed set until the last count was made. The seedling emerged each day having plumule length of $2 \mathrm{~cm}$ or more was considered as germinated.

Germination index $=\frac{\text { No.ofseedling at } 1 \text { stcount }}{\text { Daysto1 stcount }}+---++\frac{\text { No.ofseedling atfinalcount }}{\text { Daystofinalcount }}$

Seedling dry matter: The normal seedlings from each germination lot were collected and washed with running tap water. After surface drying at room temperature the seedling kept in an oven and dried at $70^{\circ} \mathrm{C}$ temperature for 72 hours and the weight express seedling ${ }^{-1}$ basis.

Data analysis was done statistically following the analysis of variance (ANOVA) technique and the means were compared by Duncan's Multiple Range Test (Gomez and Gomez, 1984).

\section{Results and Discussion}

\section{Seed moisture content (SMC)}

Storage relative humidity, initial seed moisture content and storage container had significant effect on final seed moisture content of soybean seed at 60,120, and 180 DAS (Table 1). In 2008, the lowest seed moisture content of seeds stored in 
polythene bag at $8 \%$ initial SMC in $50 \%$ RH were $8.14,8.33$, and $8.58 \%$ at 60 , 120 , and 180 DAS, respectively, and percent moisture increased 1.75, 4.13 and $7.25 \%$ while those seeds stored in cloth bag at $12 \%$ initial SMC in $80 \% \mathrm{RH}$ were $13.39,14.14$, and $14.91 \%$ at 60,120 , and 180 DAS, respectively, and \%moisture increased $11.58,17.83$ and $24.25 \%$ (Table 1). These results are in agreement with Miah (2005) who reported that soybean seeds stored at 50-60\% RH maintained moisture content less than $10 \%$ during the storage period of eight months. In 2009 , the lowest seed moisture content of seeds stored in polythene bag at $8 \%$ initial SMC in $50 \%$ RH were $8.33,8.50$, and $8.71 \%$ at 60,120 , and 180 DAS, respectively, and percent moisture increased $4.13,6.25$, and $8.87 \%$ while those seeds stored in cloth bag with $12 \%$ initial SMC at $80 \%$ RH were 13.39, 14.03, and $14.87 \%$ at 60,120 , and 180 DAS, respectively, and percent moisture increased 11.58, 16.92, and 23.92\% (Table 1). Sarmin (2009) and Locher and Bucheli (1998) also reported that seeds stored in polythene bag at lower RH showed the lower seed moisture content. Thus it appears that the moisture content of soybean seed increased with the increase of $\mathrm{RH}$ and also with increase of storage duration in both the years.

Table 1. Interaction effect of storage relative humidity, storage container and initial seed moisture content on seed moisture content of soybean seed at different days after storage in 2008 and 2009.

\begin{tabular}{|c|c|c|c|c|c|c|}
\hline \multirow{3}{*}{$\begin{array}{c}\text { Storage RH } \times \\
\text { Container } \times \\
\text { Initial SMC }\end{array}$} & \multicolumn{6}{|c|}{ Seed moisture content $(\%)$} \\
\hline & \multicolumn{3}{|c|}{2008} & \multicolumn{3}{|c|}{2009} \\
\hline & 60 DAS & 120 DAS & $180 \mathrm{DAS}$ & $60 \mathrm{DAS}$ & $120 \mathrm{DAS}$ & $180 \mathrm{DAS}$ \\
\hline $\mathrm{H}_{1} \mathrm{C}_{1} \mathrm{M}_{1}$ & $8.82 \mathrm{k}$ & 8.981 & 9.281 & $8.65 \mathrm{hi}$ & $9.04 \mathrm{~h}$ & $9.30 \mathrm{k}$ \\
\hline $\mathrm{H}_{1} \mathrm{C}_{1} \mathrm{M}_{2}$ & $12.50 \mathrm{~d}$ & $13.20 \mathrm{~d}$ & $13.82 \mathrm{~d}$ & $12.48 \mathrm{c}$ & $12.94 \mathrm{~d}$ & $13.73 \mathrm{~d}$ \\
\hline $\mathrm{H}_{1} \mathrm{C}_{2} \mathrm{M}_{1}$ & $8.14 n$ & $8.33 p$ & $8.58 \mathrm{p}$ & $8.33 \mathrm{j}$ & $8.50 \mathrm{j}$ & 8.711 \\
\hline $\mathrm{H}_{1} \mathrm{C}_{2} \mathrm{M}_{2}$ & $12.24 \mathrm{~h}$ & $12.59 \mathrm{~h}$ & $13.00 \mathrm{~h}$ & $12.13 \mathrm{~d}$ & $12.58 \mathrm{e}$ & $12.91 \mathrm{~g}$ \\
\hline $\mathrm{H}_{2} \mathrm{C}_{1} \mathrm{M}_{1}$ & $8.97 \mathrm{j}$ & $9.13 \mathrm{k}$ & $9.48 \mathrm{k}$ & $8.93 \mathrm{~g}$ & $9.15 \mathrm{hi}$ & $9.43 \mathrm{j}$ \\
\hline $\mathrm{H}_{2} \mathrm{C}_{1} \mathrm{M}_{2}$ & $12.63 \mathrm{c}$ & $13.39 \mathrm{c}$ & $14.05 \mathrm{c}$ & $12.64 \mathrm{c}$ & $13.26 \mathrm{c}$ & $13.95 \mathrm{c}$ \\
\hline $\mathrm{H}_{2} \mathrm{C}_{2} \mathrm{M}_{1}$ & $8.28 \mathrm{~m}$ & 8.540 & 8.820 & $8.42 \mathrm{ij}$ & $8.66 \mathrm{ij}$ & 8.871 \\
\hline $\mathrm{H}_{2} \mathrm{C}_{2} \mathrm{M}_{2}$ & $12.34 \mathrm{~g}$ & $12.74 \mathrm{~g}$ & $13.19 \mathrm{~g}$ & $12.35 \mathrm{~d}$ & $12.71 \mathrm{e}$ & $13.17 \mathrm{fg}$ \\
\hline $\mathrm{H}_{3} \mathrm{C}_{1} \mathrm{M}_{1}$ & $9.04 \mathrm{ij}$ & $9.24 \mathrm{j}$ & $9.64 j$ & $9.14 \mathrm{f}$ & $9.35 \mathrm{f}$ & $9.96 \mathrm{f}$ \\
\hline $\mathrm{H}_{3} \mathrm{C}_{1} \mathrm{M}_{2}$ & $12.83 b$ & $13.67 b$ & $14.87 \mathrm{~b}$ & $12.92 \mathrm{~b}$ & $13.59 \mathrm{~b}$ & $14.55 \mathrm{~b}$ \\
\hline $\mathrm{H}_{3} \mathrm{C}_{2} \mathrm{M}_{1}$ & $8.32 \mathrm{~m}$ & $8.62 n$ & $9.00 \mathrm{n}$ & $8.33 \mathrm{hi}$ & 8.69hi & $9.06 \mathrm{i}$ \\
\hline $\mathrm{H}_{3} \mathrm{C}_{2} \mathrm{M}_{2}$ & $12.50 \mathrm{f}$ & $12.95 \mathrm{f}$ & $13.62 f$ & $12.56 \mathrm{c}$ & $12.98 \mathrm{~d}$ & $13.46 \mathrm{e}$ \\
\hline $\mathrm{H}_{4} \mathrm{C}_{1} \mathrm{M}_{1}$ & $9.09 \mathrm{i}$ & $9.32 \mathrm{i}$ & $9.82 \mathrm{i}$ & $9.15 \mathrm{e}$ & $9.58 \mathrm{e}$ & $9.94 \mathrm{e}$ \\
\hline $\mathrm{H}_{4} \mathrm{C}_{1} \mathrm{M}_{2}$ & $13.39 \mathrm{a}$ & $14.14 \mathrm{a}$ & $14.91 \mathrm{a}$ & $13.36 \mathrm{a}$ & $14.03 \mathrm{a}$ & $14.87 \mathrm{a}$ \\
\hline $\mathrm{H}_{4} \mathrm{C}_{2} \mathrm{M}_{1}$ & $8.35 \mathrm{~h}$ & $8.70 \mathrm{~m}$ & $9.12 \mathrm{~m}$ & $8.87 \mathrm{gh}$ & $9.54 \mathrm{~g}$ & $10.29 \mathrm{~h}$ \\
\hline $\mathrm{H}_{4} \mathrm{C}_{2} \mathrm{M}_{2}$ & $12.59 \mathrm{e}$ & $13.45 \mathrm{e}$ & $14.01 \mathrm{e}$ & $12.89 \mathrm{~b}$ & $13.27 \mathrm{c}$ & $13.81 \mathrm{~cd}$ \\
\hline F test & $* *$ & $* *$ & $* *$ & $* *$ & $* *$ & $* *$ \\
\hline $\mathrm{CV}(\%)$ & 7.37 & 4.29 & 6.32 & 5.51 & 8.63 & 7.10 \\
\hline
\end{tabular}

DAS $=$ Days after sowing, $C V=$ Coefficient of variation, $* *=$ Significant at $1 \%$ level. In a column, figures having similar letter(s) do not differ significantly.

Note: $\mathrm{H}_{1}=50 \%$ relative humidity $(\mathrm{RH}), \mathrm{H}_{2}=60 \% \mathrm{RH}, \mathrm{H}_{3}=70 \% \mathrm{RH}, \mathrm{H}_{4}=80 \% \mathrm{RH}$,

$\mathrm{C}_{1}=$ Cloth bag, $\mathrm{C}_{2}=$ Polythene bag, $\mathrm{M}_{1}=8 \%$ Seed moisture content (SMC), $\mathrm{M}_{2}=12 \% \mathrm{SMC}$. 


\section{Germination percentage}

Storage relative humidity, initial seed moisture content and storage container had significant effect on germination percentage of soybean seed at 60, 120, and 180 DAS (Table 2). When the seed was stored, the germination was 96 and $98 \%$ in 2008 and 2009, respectively. In 2008, the highest germination percentage of seeds stored in polythene bag at $8 \%$ initial SMC with $50 \%$ RH were 93.33, 91.33, and $89.33 \%$ at 60,120 , and 180 DAS, respectively, and germination (\%) decreased

Table 2. Interaction effect of storage relative humidity, storage container and initial seed moisture content (SMC) on germination of soybean seed at different days after storage and field emergence in 2008 and 2009.

\begin{tabular}{|c|c|c|c|c|c|c|}
\hline \multirow{3}{*}{$\begin{array}{c}\text { Storage } \mathrm{RH} \times \\
\text { container } \times \\
\text { Initial SMC }\end{array}$} & \multicolumn{6}{|c|}{ Germination $(\%)$} \\
\hline & \multicolumn{3}{|c|}{2008} & \multicolumn{3}{|c|}{2009} \\
\hline & $60 \mathrm{DAS}$ & $120 \mathrm{DAS}$ & $180 \mathrm{DAS}$ & $60 \mathrm{DAS}$ & $120 \mathrm{DAS}$ & $180 \mathrm{DAS}$ \\
\hline $\mathrm{H}_{1} \mathrm{C}_{1} \mathrm{M}_{1}$ & 85.33 & $76.67 \mathrm{c}$ & $73.33 \mathrm{c}$ & $89.33 \mathrm{a}$ & $82.67 b$ & $76.67 \mathrm{~b}$ \\
\hline $\mathrm{H}_{1} \mathrm{C}_{1} \mathrm{M}_{2}$ & 72.00 & $62.00 \mathrm{~d}$ & $34.00 \mathrm{~h}$ & $72.67 b$ & $55.33 \mathrm{~cd}$ & $41.33 f g$ \\
\hline $\mathrm{H}_{1} \mathrm{C}_{2} \mathrm{M}_{1}$ & 93.33 & $91.33 \mathrm{a}$ & $89.33 a$ & $96.00 \mathrm{a}$ & $94.00 \mathrm{a}$ & $92.67 \mathrm{a}$ \\
\hline $\mathrm{H}_{1} \mathrm{C}_{2} \mathrm{M}_{2}$ & 84.00 & $75.33 c$ & $48.00 \mathrm{~g}$ & $90.00 \mathrm{a}$ & $73.33 b$ & $57.33 \mathrm{de}$ \\
\hline $\mathrm{H}_{2} \mathrm{C}_{1} \mathrm{M}_{1}$ & 81.33 & $74.67 \mathrm{c}$ & $68.00 \mathrm{~d}$ & $92.67 \mathrm{a}$ & $83.33 b$ & $73.33 b c$ \\
\hline $\mathrm{H}_{2} \mathrm{C}_{1} \mathrm{M}_{2}$ & 70.00 & $54.67 \mathrm{e}$ & $26.67 \mathrm{i}$ & $63.33 b c$ & $43.33 \mathrm{de}$ & $30.67 \mathrm{~g}$ \\
\hline $\mathrm{H}_{2} \mathrm{C}_{2} \mathrm{M}_{1}$ & 90.67 & $87.33 b$ & $85.33 b$ & $97.33 \mathrm{a}$ & $94.67 \mathrm{a}$ & $91.67 \mathrm{a}$ \\
\hline $\mathrm{H}_{2} \mathrm{C}_{2} \mathrm{M}_{2}$ & 76.67 & $62.00 \mathrm{~d}$ & $44.00 \mathrm{~g}$ & $77.33 b$ & $60.67 \mathrm{c}$ & $46.00 \mathrm{ef}$ \\
\hline $\mathrm{H}_{3} \mathrm{C}_{1} \mathrm{M}_{1}$ & 70.00 & $62.00 \mathrm{~d}$ & $52.67 f$ & $68.67 b c$ & $30.67 \mathrm{ef}$ & $0.00 \mathrm{i}$ \\
\hline $\mathrm{H}_{3} \mathrm{C}_{1} \mathrm{M}_{2}$ & 56.00 & $26.00 \mathrm{~h}$ & $0.00 \mathrm{j}$ & $66.67 b c$ & $32.00 \mathrm{ef}$ & $0.00 \mathrm{i}$ \\
\hline $\mathrm{H}_{3} \mathrm{C}_{2} \mathrm{M}_{1}$ & 81.33 & $74.00 \mathrm{c}$ & $66.00 \mathrm{~d}$ & $92.67 \mathrm{a}$ & $74.67 \mathrm{~b}$ & $65.33 \mathrm{~cd}$ \\
\hline $\mathrm{H}_{3} \mathrm{C}_{2} \mathrm{M}_{2}$ & 69.33 & $48.00 \mathrm{f}$ & $0.00 \mathrm{j}$ & $66.00 \mathrm{bc}$ & $39.33 \mathrm{e}$ & $4.00 \mathrm{~h}$ \\
\hline $\mathrm{H}_{4} \mathrm{C}_{1} \mathrm{M}_{1}$ & 66.00 & $52.00 \mathrm{ef}$ & $36.00 \mathrm{~h}$ & $54.67 \mathrm{~cd}$ & $19.33 \mathrm{~g}$ & $0.00 \mathrm{i}$ \\
\hline $\mathrm{H}_{4} \mathrm{C}_{1} \mathrm{M}_{2}$ & 48.67 & $20.00 \mathrm{i}$ & $0.00 \mathrm{j}$ & $46.00 \mathrm{~d}$ & $10.00 \mathrm{~h}$ & $0.00 \mathrm{i}$ \\
\hline $\mathrm{H}_{4} \mathrm{C}_{2} \mathrm{M}_{1}$ & 78.67 & $66.67 d$ & $58.00 \mathrm{e}$ & $94.67 \mathrm{a}$ & $73.33 b$ & $61.33 d$ \\
\hline $\mathrm{H}_{4} \mathrm{C}_{2} \mathrm{M}_{2}$ & 62.67 & $38.00 \mathrm{~g}$ & $0.00 \mathrm{j}$ & $62.00 \mathrm{bcd}$ & $23.33 \mathrm{fg}$ & $2.67 \mathrm{~h}$ \\
\hline F test & ns & $* *$ & $* *$ & $* *$ & $* *$ & $* *$ \\
\hline $\mathrm{CV}(\%)$ & 5.67 & 7.84 & 4.04 & 6.46 & 6.41 & 8.41 \\
\hline
\end{tabular}

DAS $=$ Days after sowing, $C V=$ Coefficient of variation, $n s=$ Non-significant, $* *=$ Significant at $1 \%$ level.

In a column, figures having similar letter(s) do not differ significantly.

Note: $\mathrm{H}_{1}=50 \%$ relative humidity $(\mathrm{RH}), \mathrm{H}_{2}=60 \% \mathrm{RH}, \mathrm{H}_{3}=70 \% \mathrm{RH}, \mathrm{H}_{4}=80 \% \mathrm{RH}$, $\mathrm{C}_{1}=$ Cloth bag, $\mathrm{C}_{2}=$ Polythene bag, $\mathrm{M}_{1}=8 \%$ Seed moisture content (SMC), $\mathrm{M}_{2}=12 \%$ SMC. 
$3.47,5.55$, and $6.95 \%$ while those stored in cloth bag with $12 \%$ initial SMC at $80 \%$ RH were $48.67,20.00$, and $0.00 \%$ at 60,120 , and 180 DAS, respectively, and germination (\%) decreased 51.38, 79.17, and 100.00\% (Table 2). In 2009, the highest germination percentage of seeds stored in polythene bag with $8 \%$ initial SMC at 50\% RH were $96.00,94.00$, and $92.67 \%$ at 60,120 , and 180 DAS, respectively, and germination (\%) decreased $2.04,4.08$, and $5.48 \%$ while those seeds stored in cloth bag at $12 \%$ initial SMC in $80 \%$ RH were $46.00,10.00$, and $0.00 \%$ at 60,120 , and 180 DAS, respectively, and germination (\%) decreased 53.06, 89.80, and $100.00 \%$ (Table 2). Similar decrease of soybean seedling emergence performance with increase of storage RH was also reported by Sarmin (2009).

Table 3. Interaction effect of storage relative humidity, storage container and initial seed moisture content on germination index of soybean seed at different days after storage in 2008 and 2009.

\begin{tabular}{|c|c|c|c|c|c|c|}
\hline \multirow{3}{*}{$\begin{array}{c}\text { Storage } \mathrm{RH} \times \\
\text { Container } \times \\
\text { Initial SMC }\end{array}$} & \multicolumn{6}{|c|}{ Germination index } \\
\hline & \multicolumn{3}{|c|}{2008} & \multicolumn{3}{|c|}{2009} \\
\hline & 60 DAS & 120 DAS & 180 DAS & 60 DAS & $120 \mathrm{DAS}$ & $180 \mathrm{DAS}$ \\
\hline $\mathrm{H}_{1} \mathrm{C}_{1} \mathrm{M}_{1}$ & $25.28 \mathrm{c}$ & $22.40 \mathrm{ab}$ & $13.80 \mathrm{bc}$ & $30.25 \mathrm{ab}$ & $23.13 \mathrm{ab}$ & $15.02 \mathrm{~b}$ \\
\hline $\mathrm{H}_{1} \mathrm{C}_{1} \mathrm{M}_{2}$ & $18.44 \mathrm{~g}$ & $8.84 \mathrm{~cd}$ & $4.86 \mathrm{~d}$ & 20.99de & $8.63 \mathrm{cde}$ & $5.83 \mathrm{~d}$ \\
\hline $\mathrm{H}_{1} \mathrm{C}_{2} \mathrm{M}_{1}$ & $29.53 \mathrm{a}$ & $25.04 \mathrm{a}$ & $17.81 \mathrm{a}$ & $33.25 \mathrm{a}$ & $26.04 a$ & $19.10 \mathrm{a}$ \\
\hline $\mathrm{H}_{1} \mathrm{C}_{2} \mathrm{M}_{2}$ & $23.56 \mathrm{~d}$ & $19.02 b$ & $12.35 \mathrm{c}$ & $27.93 b c$ & $21.35 b$ & $13.30 \mathrm{bc}$ \\
\hline $\mathrm{H}_{2} \mathrm{C}_{1} \mathrm{M}_{1}$ & $23.50 \mathrm{~d}$ & $18.75 b$ & $14.06 \mathrm{bc}$ & $27.43 b c$ & $23.27 \mathrm{ab}$ & $15.32 b$ \\
\hline $\mathrm{H}_{2} \mathrm{C}_{1} \mathrm{M}_{2}$ & $17.37 \mathrm{~h}$ & $11.51 \mathrm{c}$ & $4.81 \mathrm{~d}$ & $18.96 \mathrm{ef}$ & $8.86 \mathrm{~cd}$ & $4.37 \mathrm{~d}$ \\
\hline $\mathrm{H}_{2} \mathrm{C}_{2} \mathrm{M}_{1}$ & $27.30 \mathrm{~b}$ & $25.78 \mathrm{a}$ & $16.96 \mathrm{ab}$ & $31.35 \mathrm{ab}$ & $26.85 a$ & $18.59 \mathrm{a}$ \\
\hline $\mathrm{H}_{2} \mathrm{C}_{2} \mathrm{M}_{2}$ & 22.91de & $10.94 \mathrm{c}$ & $11.72 \mathrm{c}$ & $25.29 \mathrm{~cd}$ & $11.35 \mathrm{c}$ & $12.26 \mathrm{c}$ \\
\hline $\mathrm{H}_{3} \mathrm{C}_{1} \mathrm{M}_{1}$ & $22.78 \mathrm{de}$ & $8.53 \mathrm{~cd}$ & $4.20 \mathrm{de}$ & $16.88 \mathrm{ef}$ & $6.69 \mathrm{~d}-\mathrm{g}$ & $1.65 \mathrm{e}$ \\
\hline $\mathrm{H}_{3} \mathrm{C}_{1} \mathrm{M}_{2}$ & $15.19 \mathrm{i}$ & 3.36de & $0.00 \mathrm{f}$ & $16.53 \mathrm{ef}$ & $4.50 \mathrm{fgh}$ & $0.00 \mathrm{f}$ \\
\hline $\mathrm{H}_{3} \mathrm{C}_{2} \mathrm{M}_{1}$ & $25.18 \mathrm{c}$ & $20.81 \mathrm{ab}$ & $10.97 \mathrm{c}$ & $30.04 \mathrm{abc}$ & $20.27 b$ & $12.83 b c$ \\
\hline $\mathrm{H}_{3} \mathrm{C}_{2} \mathrm{M}_{2}$ & $19.76 \mathrm{f}$ & $7.28 \mathrm{~cd}$ & $1.28 \mathrm{ef}$ & $17.86 \mathrm{ef}$ & $8.00 \mathrm{c}-\mathrm{f}$ & $1.65 \mathrm{e}$ \\
\hline $\mathrm{H}_{4} \mathrm{C}_{1} \mathrm{M}_{1}$ & $20.19 f$ & $6.39 \mathrm{~cd}$ & $2.86 \mathrm{def}$ & $15.88 \mathrm{fg}$ & $3.60 \mathrm{gh}$ & $0.00 \mathrm{e}$ \\
\hline $\mathrm{H}_{4} \mathrm{C}_{1} \mathrm{M}_{2}$ & $13.89 \mathrm{j}$ & $0.700 \mathrm{e}$ & $0.00 \mathrm{f}$ & $11.34 \mathrm{~g}$ & $1.51 \mathrm{~h}$ & $0.00 \mathrm{e}$ \\
\hline $\mathrm{H}_{4} \mathrm{C}_{2} \mathrm{M}_{1}$ & $22.53 \mathrm{e}$ & $18.79 b$ & $11.31 \mathrm{c}$ & $28.98 \mathrm{abc}$ & $20.19 b$ & $12.71 b c$ \\
\hline $\mathrm{H}_{4} \mathrm{C}_{2} \mathrm{M}_{2}$ & $17.95 \mathrm{gh}$ & $7.01 \mathrm{~cd}$ & $0.00 \mathrm{f}$ & $15.86 \mathrm{fg}$ & $4.86 \mathrm{e}-\mathrm{h}$ & $0.00 \mathrm{e}$ \\
\hline $\mathrm{F}$ test & $* *$ & $* *$ & $* *$ & $* *$ & $* *$ & $* *$ \\
\hline $\mathrm{CV}(\%)$ & 5.61 & 7.10 & 7.35 & 8.49 & 4.68 & 6.04 \\
\hline
\end{tabular}

DAS= Days after sowing, $C V=$ Coefficient of variation, $* *=$ Significant at $1 \%$ level

In a column, figures having similar letter(s) do not differ significantly

Note: $\mathrm{H}_{1}=50 \%$ relative humidity (RH), $\mathrm{H}_{2}=60 \% \mathrm{RH}, \mathrm{H}_{3}=70 \% \mathrm{RH}, \mathrm{H}_{4}=80 \% \mathrm{RH}$.

$\mathrm{C}_{1}=$ Cloth bag, $\mathrm{C}_{2}=$ Polythene bag, $\mathrm{M}_{1}=8 \%$ Seed moisture content (SMC), $\mathrm{M}_{2}=12 \%$ $\mathrm{SMC}$ 


\section{Germination index}

Storage relative humidity, initial seed moisture content and storage container had significant effect on germination index of soybean seed at 60, 120, and 180 DAS (Table 3). When the seed was stored, the germination index was 31.25 and 34.80 in 2008 and 2009, respectively. In 2008, the highest germination index of seeds stored in polythene bag at $8 \%$ initial SMC in $50 \% \mathrm{RH}$ were $29.53,25.04$, and $17.81 \%$ at 60,120 and 180 DAS, respectively, and germination index decreased $5.50,19.87$, and $43.00 \%$ while those stored in cloth bag at $12 \%$ initial SMC in $80 \% \mathrm{RH}$ were $13.89,0.70$, and 0.00 at 60,120 , and 180 DAS, respectively and germination index decreased 55.55, 97.76, and $100.00 \%$ (Table 3). In 2009, the highest germination index of seeds stored in polythene bag at $8 \%$ initial SMC in $50 \%$ RH were $33.25,26.04$, and 19.10 at 60,120 , and 180 DAS, respectively, and germination index decreased $4.45,25.17$, and $45.11 \%$ while those stored in cloth bag at $12 \%$ initial SMC in $80 \%$ RH were $11.34,1.51$, and $0.00 \%$ at 60,120 , and 180 DAS, respectively, and germination index decreased 67.41, 95.66, and $100.00 \%$ (Table 3). Rahman et al. (2010) found similar result who reported that highest vigour index was obtained from 50\% RH and lowest from $80 \% \mathrm{RH}$.

\subsubsection{Seedling dry matter}

Storage relative humidity, initial seed moisture content and storage container had significant effect on seedling dry matter of soybean seed at 180 DAS (Table 4). When the seed was stored, the seedling dry matter was 0.1280 and $0.1269 / \mathrm{g}$ seedling in 2008 and 2009, respectively. In 2008, the highest seedling dry matter of seeds stored in polythene bag at $8 \%$ initial SMC in 50\% RH were 1269 , 0.1201 , and 0.1135 at 60,120 , and 180 DAS, respectively, and seedling dry matter decreased $0.86,6.17$, and $11.33 \%$ while those stored in cloth bag at $12 \%$ initial SMC in $80 \% \mathrm{RH}$ were 0.0907 and 0.0860 at 60 and 120 DAS, respectively, and seedling dry matter decreased 29.14 and $32.81 \%$ (Table 4 ). At 180 DAS, no seedling was found in those seeds stored at $80 \% \mathrm{RH}$ in cloth at $12 \%$ SMC. In 2009, the highest seedling dry matter of seeds stored in polythene bag at $8 \%$ initial SMC in 50\% RH were .1260, 0.1190, and 0.1131 at 60,120 , and 180 DAS, respectively, and seedling dry matter decreased $0.71,6.22$, and $10.87 \%$ while those stored in cloth bag at $12 \%$ initial SMC in $80 \% \mathrm{RH}$ were 0.0900 and 0.0850 at 60 and 120 DAS, respectively, and seedling dry matter decreased 29.08 and $33.02 \%$ (Table 4). At 180 DAS, no seedling was found in those seeds stored at $80 \% \mathrm{RH}$ in cloth at $12 \%$ SMC. The seedling dry matter decreased with the increase of relative humidity and initial SMC for both the years. Rahman et al. (2010) found similar result who reported that highest seedling dry matter was obtained from 50\% RH and the lowest from $80 \% \mathrm{RH}$. 
Table 4. Interaction effect of relative humidity, initial seed moisture content and container on soybean seeding dry matter of soybean at different days after storage in 2008 and 2009.

\begin{tabular}{|c|c|c|c|c|c|c|}
\hline \multirow{3}{*}{$\begin{array}{c}\text { Storage RH } \\
\times \text { Container } \\
\times \text { Initial } \\
\text { SMC }\end{array}$} & \multicolumn{6}{|c|}{ Seeding dry matter ( $\mathrm{g} /$ seedling) } \\
\hline & \multicolumn{3}{|c|}{2008} & \multicolumn{3}{|c|}{2009} \\
\hline & 60 DAS & 120 DAS & $180 \mathrm{DAS}$ & $60 \mathrm{DAS}$ & 120 DAS & $180 \mathrm{DAS}$ \\
\hline $\mathrm{H}_{1} \mathrm{C}_{1} \mathrm{M}_{1}$ & $0.1155 \mathrm{bc}$ & $0.1145 \mathrm{ab}$ & $0.1104 \mathrm{a}$ & $0.1146 b c$ & $0.1070 \mathrm{~cd}$ & $0.1000 \mathrm{c}$ \\
\hline $\mathrm{H}_{1} \mathrm{C}_{1} \mathrm{M}_{2}$ & $0.1099 \mathrm{~cd}$ & $0.1040 \mathrm{de}$ & $0.0974 \mathrm{~cd}$ & $0.1091 \mathrm{~cd}$ & $0.1032 \mathrm{de}$ & $0.0966 \mathrm{~cd}$ \\
\hline $\mathrm{H}_{1} \mathrm{C}_{2} \mathrm{M}_{1}$ & $0.1269 \mathrm{a}$ & $0.1201 \mathrm{a}$ & $0.1135 \mathrm{a}$ & $0.1260 \mathrm{a}$ & $0.1190 \mathrm{a}$ & $0.1131 \mathrm{a}$ \\
\hline $\mathrm{H}_{1} \mathrm{C}_{2} \mathrm{M}_{2}$ & $0.1146 b c$ & $0.1070 \mathrm{~cd}$ & $0.1000 \mathrm{c}$ & $0.1155 b c$ & $0.1073 \mathrm{cde}$ & $0.1030 \mathrm{c}$ \\
\hline $\mathrm{H}_{2} \mathrm{C}_{1} \mathrm{M}_{1}$ & $0.1097 \mathrm{~cd}$ & $0.1050 \mathrm{cde}$ & $0.1010 \mathrm{c}$ & $0.1113 \mathrm{def}$ & $0.1040 \mathrm{de}$ & $0.0970 \mathrm{~cd}$ \\
\hline $\mathrm{H}_{2} \mathrm{C}_{1} \mathrm{M}_{2}$ & 0.1040def & $0.1000 \mathrm{e}$ & 0.0930de & $0.1031 \mathrm{bcd}$ & $0.0991 \mathrm{e}$ & $0.0921 \mathrm{de}$ \\
\hline $\mathrm{H}_{2} \mathrm{C}_{2} \mathrm{M}_{1}$ & $0.1208 \mathrm{ab}$ & $0.1163 \mathrm{ab}$ & $0.1076 \mathrm{ab}$ & $0.1200 \mathrm{a}$ & $0.1155 \mathrm{ab}$ & $0.1065 \mathrm{ab}$ \\
\hline $\mathrm{H}_{2} \mathrm{C}_{2} \mathrm{M}_{2}$ & $0.1113 \mathrm{bcd}$ & $0.1040 \mathrm{de}$ & $0.0970 \mathrm{~cd}$ & $0.1097 \mathrm{~cd}$ & $0.1050 \mathrm{cde}$ & $0.1010 \mathrm{c}$ \\
\hline $\mathrm{H}_{3} \mathrm{C}_{1} \mathrm{M}_{1}$ & $0.1030 \mathrm{def}$ & $0.1000 \mathrm{e}$ & $0.0968 \mathrm{~cd}$ & $0.1156 f g$ & $0.1040 \mathrm{de}$ & $0.0952 b c$ \\
\hline $\mathrm{H}_{3} \mathrm{C}_{1} \mathrm{M}_{2}$ & $0.0950 \mathrm{fg}$ & $0.0905 \mathrm{fg}$ & - & $0.0943 b c$ & $0.0891 \mathrm{fg}$ & - \\
\hline $\mathrm{H}_{3} \mathrm{C}_{2} \mathrm{M}_{1}$ & $0.1158 b c$ & $0.1108 b c$ & $0.1022 b c$ & $0.1150 \mathrm{fg}$ & $0.1100 \mathrm{bc}$ & $0.1014 b c$ \\
\hline $\mathrm{H}_{3} \mathrm{C}_{2} \mathrm{M}_{2}$ & $0.1156 b c$ & $0.1040 \mathrm{de}$ & - & $0.1030 \mathrm{bc}$ & $0.1000 \mathrm{e}$ & $0.0968 \mathrm{~cd}$ \\
\hline $\mathrm{H}_{4} \mathrm{C}_{1} \mathrm{M}_{1}$ & $0.0960 \mathrm{fg}$ & $0.0930 \mathrm{f}$ & $0.0901 \mathrm{e}$ & $0.0951 \mathrm{cde}$ & $0.0920 \mathrm{f}$ & $0.0891 \mathrm{e}$ \\
\hline $\mathrm{H}_{4} \mathrm{C}_{1} \mathrm{M}_{2}$ & $0.0907 \mathrm{~g}$ & $0.0860 \mathrm{~g}$ & - & $0.0900 \mathrm{ef}$ & $0.0850 \mathrm{~g}$ & - \\
\hline $\mathrm{H}_{4} \mathrm{C}_{2} \mathrm{M}_{1}$ & 0.1069 cde & $0.1000 \mathrm{e}$ & $0.0930 \mathrm{de}$ & $0.1059 \mathrm{~g}$ & $0.0990 \mathrm{e}$ & $0.0921 \mathrm{de}$ \\
\hline $\mathrm{H}_{4} \mathrm{C}_{2} \mathrm{M}_{2}$ & $0.1000 \mathrm{ef}$ & $0.0921 \mathrm{fg}$ & - & 0.0991 & $0.0912 \mathrm{fg}$ & - \\
\hline $\mathrm{F}$ test & $* *$ & $* *$ & $* *$ & $* *$ & $* *$ & $* *$ \\
\hline $\mathrm{CV}(\%)$ & 4.70 & 6.51 & 4.68 & 4.70 & 7.51 & 4.68 \\
\hline
\end{tabular}

DAS $=$ Days after sowing, $\mathrm{CV}=$ Coefficient of variation, $* *=$ Significant at $1 \%$ level. In a column, figures having similar letter(s) do not differ significantly.

Note: $\mathrm{H}_{1}=50 \%$ relative humidity $(\mathrm{RH}), \mathrm{H}_{2}=60 \% \mathrm{RH}, \mathrm{H}_{3}=70 \% \mathrm{RH}, \mathrm{H}_{4}=80 \% \mathrm{RH}, \mathrm{C}_{1}=$ Cloth bag, $\mathrm{C}_{2}=$ Polythene bag, $\mathrm{M}_{1}=8 \%$ Seed moisture content (SMC), $\mathrm{M}_{2}=12 \%$ SMC.

\section{Conclusion}

From the result of the study, it was concluded that soybean seed could be stored with above $80 \%$ germination for at least six months under a range of relative humidities (50 to 60\%) if stored in polythene bag after drying to $8 \%$ initial seed moisture content. 


\section{References}

Agha, S. K., Z. H. Malik, M. Hatam and G. H. Jamro. 2004. Emergence of healthy seedlings of soybeans as influenced by seed storage containers. Pakistan J. Biol. Sci. 7(1): 42-44

AOSA (Association of Official Seed Analysts). 1983. Seed Vigor Testing Handbook. Contribution No. 32 to the Handbook on Seed Testing.

Chang, S. K. C., Z. S. Liu, H. J. Hou and L. A. Wilson. 2004. Influence of storage on the characteristics of soybean, soymilk and tofu. Proc. VII- World Soybean Res. Con., IV-In: Soybean Proc. and Util. Con., III- Congresso Brasileiro de Soja Brazilian Soybean Congress, Foz do Iguassu, PR, Brazil, 29 February-5 March, 977-983.

Gomez, K. A. and Gomez., A.A. 1984. Statistical procedures for Agricultural Research $2^{\text {nd }}$ Edn. John Willy and Sons., New York, Pp. 97-111.

ISTA. 2003. International Rules for Seed Testing, 2003. Zurich, Switzerland, ISTA.

Locher, R. and P. Bucheli. 1998. Comparison of soluble sugar degradation in soybean seed under simulated tropical storage conditions. Crop Sci. 38: 1229-1235.

McDonald, M. B. 1999. Seed deterioration: physiology, repair and assessment. Seed Sci. Technol. 27: 177-237.

Miah, M. A. B. 2005. Quality of soybean and wheat seed as influenced by storage relative humidity. M. S. Thesis, Dept. Agron., Bangladesh Agril. Univ., Mymensingh, Bangladesh, Pp. 25-48.

Miah, M. A. B., M. M. Rahman and M.M. Haque. 2006. Effect of relative humidity on germination and vigour of soybean seed. Inter. J. Eng. Technol. 3(1): 17-24.

O’Hare, T., J. Bagshaw and W. L. G. Johnson. 2001. Storage of oriental bunching onions. Post harvest handling of fresh vegetables. In: Proc. of a workshop held in Beijing, China, 9-11 May.

Rahman, M. M., B. Hagidok, M. M. Masood and M. N. Islam. 2010. Effect of storage container and relative humidity on the quality of wheat seed. Bangladesh J. Seed Sci. Technol. 14(1\&2): 89-94.

Sarmin, T. 2009. Effect of storage relative humidity and container on quality of shelled and unshelled groundnut seed. M.S. Thesis, Dept. Agron., Bangladesh Agril. Univ., Mymensingh, Pp. 28-65.

Tatipata, A. 2009. Effect of seed moisture content packaging and storage period on microchondria inner membrane of soybean seed. J. Agric. Technol. 5(1): 51-54.

Woodruff, M. J. 1998. Reports on the soybean: Its status, and potential for Bangladesh. Agro based Industries and Technology Development Project (ATDP). May 1998. Ministry of Agriculture and International Fertilizer Development Centre IFDC). 